\title{
Gender identity disparities in Pap test use in a sample of binary and non-binary transmasculine adults
}

\author{
Madina Agénor, ScD, MPH ${ }^{1,2}$, Jaclyn M. White Hughto, PhD, MPH',3, \\ Sarah M. Peitzmeier, PhD, MSPH ${ }^{4}$, Jennifer Potter, MD 2,5,6, Madeline B. Deutsch, MD, MPH', \\ Dana J. Pardee, $B S^{2}$, and Sari L. Reisner, SCD, $M A^{2,8,9,10}$
}

'Department of Social and Behavioral Sciences, Harvard T.H. Chan School of Public Health, Boston, MA, USA; ${ }^{2}$ The Fenway Institute, Fenway Health, Boston, MA, USA; ${ }^{3}$ Department of Epidemiology, Brown University School of Public Health, Providence, Rl, USA; ${ }^{4}$ Department of Health Behavior and Biological Sciences, University of Michigan School of Nursing, Ann Arbor, MI, USA; ${ }^{5}$ Department of Medicine, Harvard Medical School, Boston, MA, USA; 'Department of Medicine, Beth Israel Deaconess Medical Center, Boston, MA, USA; ${ }^{6}$ Department of Family and Community Medicine, University of California at San Francisco School of Medicine, San Francisco, CA, USA; ${ }^{8}$ Department of Pediatrics, Harvard Medical School, Boston, MA, USA; ${ }^{\circ}$ Department of Pediatrics, Boston Children's Hospital, Boston, MA, USA; ${ }^{10}$ Department of Epidemiology, Harvard T.H. Chan School of Public Health, Boston, MA, USA.

KEY WORDS: Cervical cancer; Screening; Transmasculine; Gender identity; Health disparities.

J Gen Intern Med 33(7):1015-7

DOI: $10.1007 / \mathrm{s} 11606-018-4400-3$

๑) Society of General Internal Medicine 2018

\section{INTRODUCTION}

Transmasculine individuals (i.e., individuals assigned female at birth who self-identify as men, transgender men, female-to-male [FTM], or another transmasculine gender identity) are at risk of developing cervical cancer $^{1}$ but face notable barriers to screening. ${ }^{2,3}$ Although transmasculine individuals are a heterogeneous population composed of persons with diverse gender identities, ${ }^{4}$ no prior study to our knowledge has examined whether cervical cancer screening differs between individuals assigned female at birth who self-identify as men, transgender men, or FTM (i.e., binary) and those who self-identify as another transmasculine gender identity such as neither exclusively male nor female, agender, or genderqueer (i.e., non-binary).

\section{METHODS}

In 2015-2016, 150 transmasculine adults living in the Greater Boston area completed a self-administered survey on their sociodemographic characteristics and sexual health. Inclusion criteria were (1) ages 21-64 years; (2) assigned female at birth; (3) self-identifies as a man, transgender man, FTM, or another transmasculine gender identity; (4) has a cervix; and (5) has had a sexual partner in the last 3 years. Participants were recruited using various strategies, including flyers, referrals from clinicians, local organizations, social media, and word of mouth.

Published online April 13, 2018
The primary predictor was gender identity, which was assessed by asking respondents to "describe [their] current gender identity." A total of 12 non-mutually exclusive categories and a fill-in-the-blank option were provided as possible responses. Participants who self-identified as men, transgender men, or FTM were categorized as having a binary gender identity, and those who self-identified as another transmasculine gender identity (e.g., genderqueer) were categorized as non-binary. The outcome was Pap test use in the last 3 years (per screening guidelines), which was created using the following question: "How long has it been since your last cervical Pap test?" Participants with missing Pap test data $(n=$ $28 ; 18.7 \%)$ were excluded from the analytic sample $(N=122)$.

We first assessed the percent distribution of Pap test use in the last 3 years among transmasculine individuals overall and tested for any difference between binary and non-binary individuals using the chi-square test. Using logistic regression, we estimated the odds ratio (OR) and $95 \%$ confidence interval (CI) for the association between gender identity and Pap test use in the last 3 years. Adjustment for age did not affect the point estimate.

\section{RESULTS}

Of 122 transmasculine adults, the majority were ages $25-$ 29 years, self-identified as white and queer, had engaged in sexual activity with cisgender (i.e., non-transgender) women and men, and had used testosterone. Most participants had a bachelor's degree or more and had private health insurance. A substantial minority of participants reported problems accessing health care in the last 12 months and avoiding care in the past year due to a fear of discrimination (Table 1). A total of $77.1 \%$ had received a Pap test in the last 3 years, with binary individuals significantly less likely than their non-binary counterparts to have been screened during that timeframe ( 71.3 vs. $96.4 \% ; p=0.004$; Table 1). Similarly, binary participants had significantly lower odds of Pap test use in the last 3 years compared to non-binary individuals (odds ratio $=0.09 ; 95 \%$ confidence interval: 0.01 , 0.71; Table 2). 


\section{DISCUSSION}

While other research has found that transmasculine individuals are less likely than cisgender women to have been screened for cervical cancer, ${ }^{2}$ we found that transmasculine individuals in our study, which included predominately white, insured, and college-educated participants, had a higher prevalence of Pap test use than US cisgender women (i.e., $69 \%$ in 2015). However, despite the elevated overall prevalence of Pap test use in our sample, our results suggest that gender identity disparities may exist among transmasculine people. In particular, binary transmasculine individuals may be significantly less likely than their non-binary peers to obtain

Table 1 Percent Distribution of Demographic, Socioeconomic, and Health Care Factors and Pap Test Use in the Last 3 Years Among Transmasculine Adults $(N=122)$

\begin{tabular}{|c|c|c|c|c|c|c|}
\hline \multirow[b]{3}{*}{ Age, continuous (years; rage: $21-50$ years) } & \multicolumn{2}{|c|}{ Total $(N=122)$} & \multicolumn{2}{|c|}{$\begin{array}{l}\text { Non-binary }(n= \\
28 ; 23.0 \%)\end{array}$} & \multicolumn{2}{|c|}{$\begin{array}{l}\text { Binary }(n=94 ; \\
77.1 \%)\end{array}$} \\
\hline & Mean & SD & Mean & SD & Mean & SD \\
\hline & 28.5 & 5.8 & 29.4 & 6.8 & 28.2 & 5.5 \\
\hline & $n$ & $\%$ & $n$ & $\%$ & $n$ & $\%$ \\
\hline Pap test in last 3 years: yes & 94 & 77.1 & 27 & 96.4 & 67 & 71.3 \\
\hline \multicolumn{7}{|l|}{ Age, categorical (years) } \\
\hline $21-24$ & 27 & 22.1 & 7 & 25.0 & 20 & 21.3 \\
\hline $25-29$ & 55 & 45.1 & 10 & 35.7 & 45 & 47.9 \\
\hline $30-34$ & 25 & 20.5 & 7 & 25.0 & 18 & 19.1 \\
\hline $35-39$ & 10 & 8.2 & 2 & 7.1 & 8 & 8.5 \\
\hline $40-50$ & 5 & 4.1 & 2 & 7.1 & 3 & 3.2 \\
\hline \multicolumn{7}{|l|}{ Race/ethnicity } \\
\hline White & 86 & 70.5 & 17 & 60.7 & 69 & 73.4 \\
\hline Black or African American & 2 & 1.6 & 0 & 0.0 & 2 & 2.1 \\
\hline Hispanic or Latinx & 13 & 10.7 & 4 & 14.3 & 9 & 9.6 \\
\hline Asian or Asian American & 8 & 6.6 & 4 & 14.3 & 4 & 4.3 \\
\hline American Indian or Alaska Native & 0 & 0.0 & 0 & 0.0 & 0 & 0.0 \\
\hline Native Hawaiian or Pacific Islander & 1 & 0.8 & 0 & 0.0 & 1 & 1.1 \\
\hline Multiracial & 12 & 9.8 & 3 & 10.7 & 9 & 9.6 \\
\hline \multicolumn{7}{|l|}{ Sexual orientation identity } \\
\hline Heterosexual & 13 & 10.7 & 0 & 0.0 & 13 & 13.8 \\
\hline Bisexual & 15 & 12.3 & 1 & 3.6 & 14 & 14.9 \\
\hline Gay or lesbian & 12 & 9.8 & 4 & 14.3 & 8 & 8.5 \\
\hline Queer & 55 & 45.1 & 13 & 46.4 & 42 & 44.7 \\
\hline Another identity ${ }^{*}$ & 27 & 22.1 & 10 & 35.7 & 17 & 18.1 \\
\hline \multicolumn{7}{|l|}{ Gender of lifetime sexual partners ${ }^{\dagger}$} \\
\hline Cisgender women & 113 & 92.6 & 27 & 96.4 & 86 & 91.5 \\
\hline Non-binary AFAB & 52 & 42.6 & 14 & 50.0 & 38 & 40.4 \\
\hline Transgender man & 48 & 39.3 & 8 & 28.6 & 40 & 42.6 \\
\hline Cisgender man & 97 & 79.5 & 20 & 71.4 & 77 & 81.9 \\
\hline Non-binary AMAB & 25 & 20.5 & 7 & 25.0 & 18 & 19.1 \\
\hline Transgender woman & 30 & 24.6 & 5 & 17.9 & 25 & 26.6 \\
\hline Lifetime history of testosterone use: yes & 99 & 81.1 & 13 & 46.4 & 86 & 91.5 \\
\hline \multicolumn{7}{|l|}{ Educational attainment } \\
\hline High school diploma or equivalent or less & 9 & 31.1 & 2 & 7.1 & 7 & 7.4 \\
\hline Some college or Associate's degree & 29 & 23.8 & 5 & 17.9 & 24 & 25.5 \\
\hline Bachelor's degree or more & 84 & 68.9 & 21 & 75.0 & 63 & 67.0 \\
\hline Student: yes & 37 & 30.3 & 7 & 25.0 & 30 & 31.9 \\
\hline \multicolumn{7}{|l|}{ Employment status $(n=119)$} \\
\hline Employed, part time & 29 & 24.4 & 10 & 37.0 & 19 & 20.7 \\
\hline Employed, full time & 30 & 25.2 & 7 & 25.9 & 23 & 25.0 \\
\hline Unemployed & 60 & 50.4 & 10 & 37.0 & 50 & 54.3 \\
\hline \multicolumn{7}{|l|}{ Annual household income $(\$ ; n=114)$} \\
\hline$\leq 32,000$ & 50 & 43.9 & 12 & 44.4 & 38 & 43.7 \\
\hline$>32,000$ & 64 & 56.1 & 15 & 55.6 & 49 & 56.3 \\
\hline \multicolumn{7}{|l|}{ Health insurance status $(n=121)$} \\
\hline Public & 41 & 33.9 & 12 & 42.9 & 29 & 31.2 \\
\hline Private & 61 & 50.4 & 10 & 35.7 & 51 & 54.8 \\
\hline Parent's & 15 & 12.4 & 3 & 10.7 & 12 & 12.9 \\
\hline Uninsured & 4 & 3.3 & 3 & 10.7 & 1 & 1.1 \\
\hline Any problem accessing health care in last 12 months: yes ${ }^{\ddagger}(n=120)$ & 15 & 12.3 & 4 & 14.3 & 11 & 11.7 \\
\hline Avoided health care in last 12 months due to fear of discrimination: yes & 23 & 18.9 & 6 & 21.4 & 17 & 18.1 \\
\hline
\end{tabular}

Percentages are based on column totals

Queer includes individuals who self-identified as queer, pansexual, and other non-heterosexual identities. Proportions (\%) may not add to $100.0 \%$ due to rounding error

AFAB: assigned female at birth; AMAB: assigned male at birth

*Includes pansexual, asexual, questioning, no label, homoflexible, and stud

†Categories are not mutually exclusive

tRestricted to those who sought health care in the last 12 months 
Table 2 Odds of Pap Test Use in the Last 3 Years in Relation to Gender Identity Among Transmasculine Adults $(N=122)$

\begin{tabular}{lll}
\hline \hline & OR & $\mathbf{9 5 \% ~ C I ~}$ \\
\hline Gender identity & & \\
Non-binary (reference) & 1.00 & - \\
Binary & 0.09 & $0.01-0.71$ \\
\hline
\end{tabular}

Values in italics indicate statistically significant odds ratio (OR) and 95\% confidence interval (CI) at the 0.05 level

regular Pap tests. This disparity may be explained by Pap testing barriers that are more pronounced among binary transmasculine individuals relative to their non-binary counterparts, including transphobia, ${ }^{4}$ lack of health insurance, ${ }^{3}$ provider discomfort, ${ }^{5}$ and patient distress associated with the dissonance between one's anatomy and gender identity as a man, transgender man, or FTM. ${ }^{6}$ Additional research is needed to identify the drivers of gender identity disparities in Pap test use among transmasculine individuals, as well as between transmasculine individuals and cisgender women, ${ }^{2}$ in order to inform evidence-based interventions that promote equity in cervical cancer screening, both across and within gender identity groups.

Acknowledgements: We are grateful to the transmasculine individuals who participated in this study. We would also like to thank the study Task Force (Tre'Andre Valentine, Mason Dunn, Landen Motyka, Thomas Lewis, Ruben Hopwood, MDiv, PhD, Yvonne Gomez-Carrion, $M D$, Joshua Safer, MD, FACP, Julie Thompson, PA-C, Van Bailey, EdD, and Elizabeth Boskey, PhD) and study providers (Timothy Cavanaugh, MD, Tracey Toner, NP, Ellie Doig, NP, Ryan Tappin, NP, and Jessica Piccirilli, PA).
Corresponding Author: Madina Agénor, ScD, MPH; Department of Social and Behavioral Sciences, Harvard T.H. Chan School of Public Health, Bonton, MA, USA (e-mail: magenor@hsph.harvard.edu).

Funding information This research was funded by a PatientCentered Outcomes Research Institute (PCORI) grant awarded to Dr. Sari Reisner (CER-1403-12625; CinicalTrials.gov Identifier: NCT02401867).

Compliance with ethical standards: This study was approved by the Fenway Health Institutional Review Board.

Conflict of Interest: The authors declare that they do not have any conflict of interest.

\section{REFERENCES}

1. Reisner SL, Murchison G. A global research synthesis of HIV and STI biobehavioural risks in female-to-male transgender adults. Glob Public Health 2016;11:866-887.

2. Tabaac AR, Sutter ME, Wall CS, Baker KE. Gender identity disparities in cancer screening behaviors. Am J Prev Med 2018. DOI: https://doi.org/ 10.1016/j.amepre.2017.11.009.

3. Potter J, Peitzmeier SM, Bernstein I, Reisner SL, Alizaga NM, Agénor M, Pardee DJ. Cervical cancer screening for patients on the female-tomale spectrum: a narrative review and guide for clinicians. J Gen Intern Med 2015;30:1857-1864.

4. James SE, Herman JL, Rankin S, Keisling M, Mottet L, Anafi M. The report of the 2015 U.S. Transgender Survey. Washington, DC: National Center for Transgender Equality. 2016. Available at: http://www. transequality.org/sites/default/files/docs/USTS-Full-Report-FINAL.PDF. Accessed November 1, 2017.

5. Unger CA. Care of the transgender patient: a survey of gynecologists' current knowledge and practice. J Womens Health 2015;24:114-118.

6. Peitzmeier SM, Agénor M, Bernstein IM, McDowell M, Alizaga NM, Reisner SL, Pardee DJ, Potter J. "It can promote an existential crisis:" factors influencing Pap test acceptability and utilization among transmasculine individuals. Qual Health Res 2017;27:2138-2149. 